\title{
Strong convergence of a modified iterative algorithm for hierarchical fixed point problems and variational inequalities
}

Yuanheng Wang ${ }^{1 *}$ and Wei $\mathrm{Xu}^{2}$

\section{"Correspondence:}

wangyuanheng@yahoo.com.cn

1 Department of Mathematics,

Zhejiang Normal University,

Zhejiang, 321004, China

\begin{abstract}
This article aims to deal with a new modified iterative projection method for solving a hierarchical fixed point problem. It is shown that under certain approximate assumptions of the operators and parameters, the modified iterative sequence $\left\{x_{n}\right\}$ converges strongly to a fixed point $x^{*}$ of $T$, also the solution of a variational inequality. As a special case, this projection method solves some quadratic minimization problem. The results here improve and extend some recent corresponding results by other authors.
\end{abstract}

MSC: 47H10; 47J20; 47H09; 47H05

Keywords: hierarchical fixed point; nonexpansive mapping; Lipschitzian and strongly monotone mapping; quadratic minimization; modified iterative projection algorithm

\section{Introduction}

Let $\Omega$ be a nonempty closed convex subset of a real Hilbert space $H$ with the inner product $\langle\cdot, \cdot\rangle$ and the norm $\|\cdot\|$. Recall that a mapping $T: \Omega \longmapsto H$ is called $L$-Lipschitzian if there exits a constant $L$ such that $\|T x-T y\| \leq L\|x-y\|, \forall x, y \in \Omega$. In particular, if $L \in[0,1)$, then $T$ is said to be a contraction; if $L=1$, then $T$ is called a nonexpansive mapping. We denote by $\operatorname{Fix}(T)$ the set of the fixed points of $T$, i.e., $\operatorname{Fix}(T)=\{x \in \Omega: T x=x\}$.

A mapping $F: \Omega \longmapsto H$ is called $\eta$-strongly monotone if there exists a constant $\eta \geq 0$ such that

$$
\langle x-y, F x-F y\rangle \geq \eta\|x-y\|^{2}, \quad \forall x, y \in \Omega .
$$

In particular, if $\eta=0$, then $F$ is said to be monotone.

A mapping $P_{\Omega}: H \longmapsto \Omega$ is called a metric projection if there exists a unique nearest point in $\Omega$ denoted by $P_{\Omega} x$ such that

$$
\left\|x-P_{\Omega} x\right\|=\inf _{y \in \Omega}\|x-y\|, \quad \forall x \in H .
$$

Recently many authors investigated the fixed point problem of nonexpansive mappings, generalized nonexpansive mappings with C-conditions, a family of finite or infinite nonexpansive mappings and pseudo-contractions and obtained many useful results; see, for example, $[1-12]$ and the references therein.

(c) 2013 Wang and Xu; licensee Springer. This is an Open Access article distributed under the terms of the Creative Commons Attribution License (http://creativecommons.org/licenses/by/2.0), which permits unrestricted use, distribution, and reproduction in any medium, provided the original work is properly cited. 
Now, we focus on the following problem.

To find a hierarchical fixed point of $T$ with respect to another operator $S$ is to find an $x^{*} \in \operatorname{Fix}(T)$ satisfying

$$
\left\langle x^{*}-S x^{*}, x^{*}-x\right\rangle \leq 0, \quad \forall x \in \operatorname{Fix}(T)
$$

which is equivalent to the following fixed point problem: to find an $x^{*} \in \Omega$ that satisfies $x^{*}=P_{\mathrm{Fix}(T)} S x^{*}$. We know that $\operatorname{Fix}(T)$ is closed and convex, so the metric projection $P_{\mathrm{Fix}(T)}$ is well defined.

It is well known that the iterative methods for finding hierarchical fixed points of nonexpansive mappings can also be used to solve a convex minimization problem; see, for example, [13,14] and the references therein. In 2006, Marino and Xu [15] considered the following general iterative method:

$$
x_{n+1}=\alpha_{n} \gamma f\left(x_{n}\right)+\left(I-\alpha_{n} A\right) T x_{n}, \quad n \geq 0,
$$

where $f$ is a contraction, $T$ is a nonexpansive mapping, $A$ is a bounded linear strongly positive operator: $\langle A x, x\rangle \geq \zeta\|x\|^{2}, \forall x \in H$, for some $\zeta>0$. And it is proved that if the sequence $\left\{\alpha_{n}\right\}$ of parameters satisfies appropriate conditions, then the sequence $\left\{x_{n}\right\}$ generated by (2) converges strongly to the unique solution of the variational inequality

$$
\left\langle(\gamma f-A) x^{*}, x-x^{*}\right\rangle \leq 0, \quad \forall x \in C \in H
$$

which is the optimality condition for the minimization problem

$$
\min _{x \in C}\left\{\frac{1}{2}\langle A x, x\rangle-h(x)\right\}
$$

where $h$ is a potential function for $\gamma f$, i.e., $h^{\prime}(x)=\gamma f(x), \forall x \in H$.

In 2010, Tian [16] introduced the general steepest-descent method

$$
x_{n+1}=\alpha_{n} \rho f\left(x_{n}\right)+\left(I-\alpha_{n} \mu F\right) T x_{n}, \quad \forall n \geq 0,
$$

where $F$ is an $L$-Lipschitzian and $\eta$-strongly monotone operator. Under certain approximate conditions, the sequence $\left\{x_{n}\right\}$ generated by (3) converges strongly to a fixed point of $T$, which solves the variational inequality

$$
\left\langle(\rho f-\mu F) x^{*}, x-x^{*}\right\rangle \leq 0, \quad \forall x \in \operatorname{Fix}(T) .
$$

Very recently, Ceng et al. [17] investigated the following iterative method:

$$
x_{n+1}=P_{\Omega}\left[\alpha_{n} \rho U x_{n}+\left(I-\alpha_{n} \mu F\right) T x_{n}\right], \quad \forall n \geq 0,
$$

where $U$ is a Lipschitzian (possibly non-self) mapping, and $F$ is a Lipschitzian and strongly monotone mapping. They proved that under some approximate assumptions on the operators and parameters, the sequence $\left\{x_{n}\right\}$ generated by (4) converges strongly to the unique 
solution of the variational inequality

$$
\left\langle(\rho U-\mu F) x^{*}, x-x^{*}\right\rangle \leq 0, \quad \forall x \in \operatorname{Fix}(T) .
$$

On the other hand, in 2010, Yao et al. [18] investigated an iterative method for a hierarchical fixed point problem by

$$
\left\{\begin{array}{l}
y_{n}=\beta_{n} S x_{n}+\left(1-\beta_{n}\right) x_{n}, \\
x_{n+1}=P_{\Omega}\left[\alpha_{n} f\left(x_{n}\right)+\left(1-\alpha_{n}\right) T y_{n}\right], \quad \forall n \geq 0,
\end{array}\right.
$$

where $S: \Omega \longmapsto \Omega$ is a nonexpansive mapping. Under some approximate assumptions of the parameters, the sequence $\left\{x_{n}\right\}$ generated by (6) converges strongly to the unique solution of the variational inequality

$$
x^{*} \in F(T), \quad\left\langle(I-f) x^{*}, x-x^{*}\right\rangle \geq 0, \quad \forall x \in \operatorname{Fix}(T) .
$$

Motivated and inspired by the above research work, we introduce the following modified iterative method for a hierarchical fixed point problem:

$$
\left\{\begin{array}{l}
y_{n}=\beta_{n} S x_{n}+\left(1-\beta_{n}\right) x_{n}, \\
x_{n+1}=P_{\Omega}\left[\alpha_{n} \rho U\left(x_{n}\right)+\left(I-\alpha_{n} \mu F\right) T y_{n}, \quad \forall n \geq 0,\right.
\end{array}\right.
$$

where $S, T$ are nonexpansive mappings with $\operatorname{Fix}(T) \neq \emptyset, U$ is a $\gamma$-Lipschitzian (possibly non-self) mapping, $F$ is an $L$-Lipschitzian and $\eta$-strongly monotone operator. We prove that the sequence $\left\{x_{n}\right\}$ generated by (7) converges strongly to the unique solution of the variational inequality (5) if the operators and parameters satisfy some approximate conditions. As a special case, this projection method also solves the quadratic minimization problem $x_{n} \rightarrow \operatorname{argmin}_{x \in \operatorname{Fix}(T)}\|x\|^{2}$.

Obviously, (2), (3), (4) and (6) are some special cases of (7), respectively. So, our results improve and extend many recent corresponding results of other authors such as $[5,13$, $15-19]$.

\section{Preliminaries}

This section contains some lemmas which will be used in the proofs of our main results in the following section.

Lemma 2.1 [18] Let $x \in H$ and $z \in \Omega$ be any points. The following results hold.

(1) $P_{\Omega}: H \rightarrow \Omega$ is nonexpansive and $z=P_{\Omega} x$ if and only if the following relation holds:

$$
\langle x-z, y-z\rangle \leq 0, \quad \forall y \in \Omega
$$

(2) $z=P_{\Omega} x$ if and only if the following relation holds:

$$
\|x-z\|^{2} \leq\|x-y\|^{2}-\|y-z\|^{2}, \quad \forall y \in \Omega .
$$


Lemma 2.2 [4] Let $H$ be a real Hilbert space, $\forall x, y \in H$, the following inequality holds:

$$
\|x+y\|^{2} \leq\|x\|^{2}+2\langle y, x+y\rangle .
$$

Lemma 2.3 [17] Let $U: \Omega \longmapsto H$ be a $\gamma$-Lipschitzian mapping with a constant $\gamma \geq 0$ and let $F: \Omega \longmapsto H$ be a $k$-Lipschitzian and $\eta$-strongly monotone mapping with constants $k, \eta>0$, then for $0 \leq \rho \gamma<\mu \eta$,

$$
\langle x-y,(\mu F-\rho U) x-(\mu F-\rho U) y\rangle \geq(\mu \eta-\rho \gamma)\|x-y\|^{2}, \quad \forall x, y \in \Omega .
$$

That is to say, the operator $\mu F-\rho U$ is $\mu \eta-\rho \gamma$-strongly monotone.

Lemma 2.4 [10] (Demiclosedness principle) Let $\Omega$ be a nonempty closed convex subset of a real Hilbert space $H$ and let $T: \Omega \longmapsto \Omega$ be a nonexpansive mapping with $\operatorname{Fix}(T) \neq \emptyset$. If $\left\{x_{n}\right\}$ is a sequence in $\Omega$ weakly converging to $x$ and $\left\{(I-T) x_{n}\right\}$ converges strongly to $y$, then $(I-T) x=y$. In particular, if $y=0$, then $x \in F(T)$.

Lemma 2.5 [19] Suppose that $\lambda \in(0,1)$ and $\mu>0$. Let $F: \Omega \longmapsto H$ be an L-Lipschitzian and $\eta$-strongly monotone operator with constants $L, \eta>0$. In association with a nonexpansive mapping $T: \Omega \longmapsto \Omega$, define the mapping $T^{\lambda}: \Omega \longmapsto H$ by

$$
T^{\lambda} x:=T x-\lambda \mu F T(x), \quad \forall x \in \Omega
$$

Then $T^{\lambda}$ is a contraction provided $\mu<\frac{2 \eta}{L^{2}}$, that is,

$$
\left\|T^{\lambda} x-T^{\lambda} y\right\| \leq(1-\lambda v)\|x-y\|, \quad \forall x \in \Omega,
$$

where $v=1-\sqrt{1-\mu\left(2 \eta-\mu L^{2}\right)}$.

Lemma 2.6 [20] Let $\left\{\alpha_{n}\right\}$ be a sequence of nonnegative real numbers satisfying the following relation:

$$
\alpha_{n+1} \leq\left(1-\gamma_{n}\right) \alpha_{n}+\delta_{n}
$$

with conditions

(1) $\left\{\gamma_{n}\right\} \subset(0,1), \Sigma_{n=1}^{\infty} \gamma_{n}=\infty$;

(2) $\lim \sup _{n \rightarrow \infty} \frac{\delta_{n}}{\gamma_{n}}=0$ or $\Sigma_{n=1}^{\infty}\left|\delta_{n}\right|<\infty$.

Then $\lim _{n \rightarrow \infty} \alpha_{n}=0$.

\section{Main results}

Theorem 3.1 Let $\Omega$ be a nonempty closed convex subset of a real Hilbert space $H$ and let $x_{0} \in \Omega$ be any given initial guess. Let $S, T: \Omega \longmapsto \Omega$ be nonexpansive mappings such that $\operatorname{Fix}(T) \neq \emptyset$. Let $F: \Omega \longmapsto H$ be an L-Lipschitzian and $\eta$-strongly monotone (possibly non-self) operator with coefficients $L, \eta>0$. Let $U: \Omega \longmapsto H$ be a $\gamma$-Lipschitzian (possibly non-self) mapping with a coefficient $\gamma \geq 0$. Suppose the parameters satisfy $0<\mu<\frac{2 \eta}{L^{2}}$, $0 \leq \rho \gamma<v$, where $v=1-\sqrt{1-\mu\left(2 \eta-\mu L^{2}\right)}$. And suppose the sequences $\left\{\alpha_{n}\right\},\left\{\beta_{n}\right\} \subset[0,1]$ satisfy the following conditions: 
(i) $\lim _{n \rightarrow \infty} \alpha_{n}=0$ and $\Sigma_{n=0}^{\infty} \alpha_{n}=\infty$;

(ii) $\lim _{n \rightarrow \infty} \frac{\beta_{n}}{\alpha_{n}}=0$;

(iii) $\sum_{n=0}^{\infty}\left|\alpha_{n+1}-\alpha_{n}\right|<\infty$ and $\sum_{n=0}^{\infty}\left|\beta_{n+1}-\beta_{n}\right|<\infty$.

Then the sequence $\left\{x_{n}\right\}$ generated by (7) converges strongly to a fixed point $x^{*}$ of $T$, which is the unique solution of the variational inequality (5). In particular, if we take $U=0$, $F=I$, then $x_{n}$ defined by (7) converges in norm to the minimum norm fixed point $x^{*}$ of $T$, namely, the point $x^{*}$ is the unique solution to the quadratic minimization problem $x^{*}=\operatorname{argmin}_{x \in \operatorname{Fix}(T)}\|x\|^{2}$.

Proof We divide the proof into six steps.

Step 1 . We first show that the variational inequality (5) has only one solution. Observe that the constants satisfy $0 \leq \rho \gamma<v$ and

$$
\begin{aligned}
L \geq \eta & \Longleftrightarrow L^{2} \geq \eta^{2} \\
& \Longleftrightarrow 1-2 \mu \eta+\mu^{2} L^{2} \geq 1-2 \mu \eta+\mu^{2} \eta^{2} \\
& \Longleftrightarrow \sqrt{1-\mu\left(2 \eta-\mu L^{2}\right)} \geq 1-\mu \eta \\
& \Longleftrightarrow \mu \eta \geq 1-\sqrt{1-\mu\left(2 \eta-\mu L^{2}\right)} \\
& \Longleftrightarrow \mu \eta \geq v,
\end{aligned}
$$

therefore the operator $\mu F-\rho U$ is $\mu \eta-\rho \gamma$-strongly monotone, and we get the uniqueness of the solution of the variational inequality (5) and denote it by $x^{*} \in \operatorname{Fix}(T)$.

Step 2. Then we get that the sequences $\left\{x_{n}\right\}$ and $\left\{y_{n}\right\}$ are bounded. By condition (ii), without loss of generality, we may assume $\beta_{n} \leq \alpha_{n}, \forall n \geq 0$. Taking a fixed point $p \in \operatorname{Fix}(T)$, we have

$$
\begin{aligned}
\left\|y_{n}-p\right\| & =\left\|\beta_{n} S x_{n}+\left(1-\beta_{n}\right) x_{n}-p\right\| \\
& \leq \beta_{n}\left\|S x_{n}-S p\right\|+\beta_{n}\|S p-p\|+\left(1-\beta_{n}\right)\left\|x_{n}-p\right\| \\
& \leq\left\|x_{n}-p\right\|+\beta_{n}\|S p-p\| .
\end{aligned}
$$

On the other hand, denoting $V_{n}=\alpha_{n} \rho U x_{n}+\left(I-\alpha_{n} \mu F\right) T y_{n}$, from (7) we get

$$
\begin{aligned}
\left\|x_{n+1}-p\right\| & =\left\|P_{\Omega}\left(V_{n}\right)-P_{\Omega} p\right\| \\
& \leq\left\|\alpha_{n} \rho U x_{n}+\left(I-\alpha_{n} \mu F\right) T y_{n}-p\right\| \\
& \leq \alpha_{n}\left\|\rho U x_{n}-\mu F p\right\|+\left\|\left(I-\alpha_{n} \mu F\right) T y_{n}-\left(I-\alpha_{n} \mu F\right) T p\right\| \\
& \leq \alpha_{n} \rho \gamma\left\|x_{n}-p\right\|+\alpha_{n}\|(\rho U-\mu F) p\|+\left(1-\alpha_{n} \nu\right)\left\|y_{n}-p\right\| .
\end{aligned}
$$

Together with (8) and (9), we have

$$
\begin{aligned}
\left\|x_{n+1}-p\right\| \leq & \alpha_{n} \rho \gamma\left\|x_{n}-p\right\|+\left(1-\alpha_{n} \nu\right)\left\|x_{n}-p\right\| \\
& +\alpha_{n}\|(\rho U-\mu F) p\|+\left(1-\alpha_{n} \nu\right) \beta_{n}\|S p-p\| \\
\leq & {\left[1-\alpha_{n}(\nu-\rho \gamma)\right]\left\|x_{n}-p\right\|+\alpha_{n}[\|(\rho U-\mu F) p\|+\|S p-p\|] }
\end{aligned}
$$




$$
\begin{aligned}
= & {\left[1-\alpha_{n}(\nu-\rho \gamma)\right]\left\|x_{n}-p\right\| } \\
& +\alpha_{n}(\nu-\rho \gamma)\left[\frac{1}{v-\rho \gamma}(\|(\rho U-\mu F) p\|+\|S p-p\|)\right] .
\end{aligned}
$$

Hence

$$
\left\|x_{n}-p\right\| \leq \max \left\{\left\|x_{0}-p\right\|, \frac{1}{v-\rho \gamma}(\|(\rho U-\mu F) p\|+\|S p-p\|)\right\} .
$$

We get the sequence $\left\{x_{n}\right\}$ is bounded, and so are $\left\{y_{n}\right\},\left\{S x_{n}\right\},\left\{T x_{n}\right\},\left\{F T y_{n}\right\}\left\{U x_{n}\right\}$.

Step 3. Next we show that $\left\|x_{n+1}-x_{n}\right\| \rightarrow 0$ as $n \rightarrow \infty$. Estimate $\left\|y_{n}-y_{n-1}\right\|$

$$
\begin{aligned}
\left\|y_{n}-y_{n-1}\right\|= & \left\|\beta_{n} S x_{n}-\left(1-\beta_{n}\right) x_{n}-\left(\beta_{n-1} S x_{n-1}-\left(1-\beta_{n-1}\right) x_{n-1}\right)\right\| \\
\leq & \beta_{n}\left\|S x_{n}-S x_{n-1}\right\|+\left(1-\beta_{n}\right)\left\|x_{n}-x_{n-1}\right\| \\
& +\left|\beta_{n}-\beta_{n-1}\right|\left(\left\|S x_{n-1}\right\|+\left\|x_{n-1}\right\|\right) \\
\leq & \left\|x_{n}-x_{n-1}\right\|+\left|\beta_{n}-\beta_{n-1}\right| M,
\end{aligned}
$$

where $\mathrm{M}$ is a constant such that

$$
\begin{aligned}
\sup _{n \geq 1}\left\{\left\|S x_{n}\right\|+\right. & \left.\left\|x_{n}\right\|+\rho\left\|U x_{n}+\right\| \mu F T y_{n}\|+\| S x^{*}-x^{*}\|\cdot\| x_{n+1}-x^{*} \|\right\} \leq M, \\
\left\|x_{n+1}-x_{n}\right\|= & \left\|P_{\Omega}\left(V_{n}\right)-P_{\Omega}\left(V_{n-1}\right)\right\| \\
\leq & \| \alpha_{n} \rho\left(U x_{n}-U x_{n-1}\right)+\left(\alpha_{n}-\alpha_{n-1}\right) \rho U x_{n-1}+\left(I-\alpha_{n} \mu F\right) T y_{n} \\
& \quad-\left(I-\alpha_{n} \mu F\right) T y_{n-1}+\left(I-\alpha_{n} \mu F\right) T y_{n-1}-\left(I-\alpha_{n-1} \mu F\right) T y_{n-1} \| \\
\leq & \alpha_{n} \rho \gamma\left\|x_{n}-x_{n-1}\right\|+\left(1-\alpha_{n} v\right)\left\|y_{n}-y_{n-1}\right\| \\
& +\left|\alpha_{n}-\alpha_{n-1}\right|\left(\left\|\rho U x_{n-1}\right\|+\left\|\mu F T y_{n-1}\right\|\right) .
\end{aligned}
$$

Substituting (10) into (11), we obtain

$$
\begin{aligned}
\left\|x_{n+1}-x_{n}\right\| \leq & \alpha_{n} \rho \gamma\left\|x_{n}-x_{n-1}\right\|+\left(1-\alpha_{n} v\right)\left\|x_{n}-x_{n-1}\right\|+\left(1-\alpha_{n} v\right)\left|\beta_{n}-\beta_{n-1}\right| M \\
& +\left|\alpha_{n}-\alpha_{n-1}\right|\left(\left\|\rho U x_{n-1}\right\|+\left\|\mu F T y_{n-1}\right\|\right) \\
\leq & {\left[1-\alpha_{n}(\nu-\rho \gamma)\right]\left\|x_{n}-x_{n-1}\right\|+\left(\left|\alpha_{n}-\alpha_{n-1}\right|+\left|\beta_{n}-\beta_{n-1}\right|\right) M . }
\end{aligned}
$$

Notice the conditions (i) and (iii), by Lemma 2.6, we have $\left\|x_{n+1}-x_{n}\right\| \rightarrow 0$ as $n \rightarrow \infty$.

Step 4. Next we show that $\left\|x_{n}-T x_{n}\right\| \rightarrow 0$ as $n \rightarrow \infty$.

$$
\begin{aligned}
\left\|x_{n}-T x_{n}\right\| & \leq\left\|x_{n}-x_{n+1}\right\|+\left\|x_{n+1}-T x_{n}\right\| \\
& \leq\left\|x_{n}-x_{n+1}\right\|+\left\|\operatorname{Proj}_{\Omega}\left(V_{n}\right)-\operatorname{Proj}_{\Omega} T x_{n}\right\| \\
& \leq\left\|x_{n}-x_{n+1}\right\|+\left\|\alpha_{n}\left(\rho U x_{n}-\mu F T y_{n}\right)+T y_{n}-T x_{n}\right\| \\
& \leq\left\|x_{n}-x_{n+1}\right\|+\alpha_{n}\left\|\rho U x_{n}-\mu F T y_{n}\right\|+\left\|y_{n}-x_{n}\right\| \\
& \leq\left\|x_{n}-x_{n+1}\right\|+\alpha_{n}\left\|\rho U x_{n}-\mu F T y_{n}\right\|+\beta_{n}\left\|S x_{n}-x_{n}\right\| .
\end{aligned}
$$


Notice that $\alpha_{n} \rightarrow 0, \beta_{n} \rightarrow 0,\left\|\rho U x_{n}-\mu F T y_{n}\right\|$ and $\left\|S x_{n}-x_{n}\right\|$ are bounded, and we have $\left\|x_{n}-T x_{n}\right\| \rightarrow 0$ as $n \rightarrow \infty$.

Step 5. Now we show that $\lim _{\sup } \operatorname{su}_{n \rightarrow \infty}\left\langle(\rho U-\mu F) x^{*}, x_{n}-x^{*}\right\rangle \leq 0$, where $x^{*}$ is the unique solution of the variational inequality. Since $\left\{x_{n}\right\}$ is bounded, we take a subsequence $\left\{x_{n_{k}}\right\}$ of $\left\{x_{n}\right\}$ such that

$$
\limsup _{n \rightarrow \infty}\left\langle(\rho U-\mu F) x^{*}, x_{n}-x^{*}\right\rangle=\limsup _{k \rightarrow \infty}\left\langle(\rho U-\mu F) x^{*}, x_{n_{k}}-x^{*}\right\rangle,
$$

and we assume $x_{n_{k}} \rightarrow x^{\prime}$. By Lemma 2.4, we have $x^{\prime} \in \operatorname{Fix}(T)$. Therefore

$$
\limsup _{n \rightarrow \infty}\left\langle(\rho U-\mu F) x^{*}, x_{n}-x^{*}\right\rangle=\left\langle(\rho U-\mu F) x^{*}, x^{\prime}-x^{*}\right\rangle \leq 0 .
$$

Hence

$$
\begin{aligned}
\left\|x_{n+1}-x^{*}\right\|^{2}= & \left\langle P_{\Omega}\left(V_{n}\right)-x^{*}, x_{n+1}-x^{*}\right\rangle \\
= & \left\langle P_{\Omega}\left(V_{n}\right)-V_{n}, P_{\Omega}\left(V_{n}\right)-x^{*}\right\rangle+\left\langle V_{n}-x^{*}, x_{n+1}-x^{*}\right\rangle \\
\leq & \left\langle\alpha_{n}\left(\rho U x_{n}-\mu F x^{*}\right)+\left(I-\alpha_{n} \mu F\right) T y_{n}-\left(I-\alpha_{n} \mu F\right) T x^{*}, x_{n+1}-x^{*}\right\rangle \\
= & \alpha_{n} \rho\left\langle U x_{n}-U x^{*}, x_{n+1}-x^{*}\right\rangle+\alpha_{n}\left\langle\rho U x^{*}-\mu F x^{*}, x_{n+1}-x^{*}\right\rangle \\
& +\left\langle\left(I-\alpha_{n} \mu F\right) T y_{n}-\left(I-\alpha_{n} \mu F\right) T x^{*}, x_{n+1}-x^{*}\right\rangle \\
\leq & \alpha_{n} \rho \gamma\left\|x_{n}-x^{*}\right\| \cdot\left\|x_{n+1}-x^{*}\right\|+\alpha_{n}\left\langle\rho U x^{*}-\mu F x^{*}, x_{n+1}-x^{*}\right\rangle \\
& +\left(1-\alpha_{n} v\right)\left\|y_{n}-x^{*}\right\| \cdot\left\|x_{n+1}-x^{*}\right\| .
\end{aligned}
$$

On the other hand, taking $p=x^{*}$ in (8), we obtain $\left\|y_{n}-x^{*}\right\| \leq\left\|x_{n}-x^{*}\right\|+\beta_{n}\left\|S x^{*}-x^{*}\right\|$. Together with (12), we have

$$
\begin{aligned}
\left\|x_{n+1}-x^{*}\right\|^{2} \leq & \alpha_{n} \rho \gamma\left\|x_{n}-x^{*}\right\| \cdot\left\|x_{n+1}-x^{*}\right\|+\alpha_{n}\left\langle\rho U x^{*}-\mu F x^{*}, x_{n+1}-x^{*}\right\rangle \\
& +\left(1-\alpha_{n} v\right)\left(\left\|x_{n}-x^{*}\right\|+\beta_{n}\left\|S x^{*}-x^{*}\right\|\right) \cdot\left\|x_{n+1}-x^{*}\right\| \\
= & {\left[1-\alpha_{n}(v-\rho \gamma)\right]\left\|x_{n}-x^{*}\right\| \cdot\left\|x_{n+1}-x^{*}\right\|+\alpha_{n}\left(\rho U x^{*}-\mu F x^{*}, x_{n+1}-x^{*}\right\rangle } \\
& +\left(1-\alpha_{n} \nu\right) \beta_{n}\left\|S x^{*}-x^{*}\right\| \cdot\left\|x_{n+1}-x^{*}\right\| \\
\leq & \frac{1-\alpha_{n}(v-\rho \gamma)}{2}\left(\left\|x_{n}-x^{*}\right\|^{2}+\left\|x_{n+1}-x^{*}\right\|^{2}\right) \\
& +\alpha_{n}\left\langle\rho U x^{*}-\mu F x^{*}, x_{n+1}-x^{*}\right\rangle \\
& +\beta_{n}\left\|S x^{*}-x^{*}\right\| \cdot\left\|x_{n+1}-x^{*}\right\|,
\end{aligned}
$$

which implies that

$$
\begin{aligned}
\left\|x_{n+1}-x^{*}\right\|^{2} \leq & \frac{1-\alpha_{n}(v-\rho \gamma)}{1+\alpha_{n}(v-\rho \gamma)}\left\|x_{n}-x^{*}\right\|^{2} \\
& +\frac{2}{1+\alpha_{n}(v-\rho \gamma)}\left[\alpha_{n}\left\langle\rho U x^{*}-\mu F x^{*}, x_{n+1}-x^{*}\right\rangle+\beta_{n} M\right] \\
\leq & {\left[1-\alpha_{n}(v-\rho \gamma)\right]\left\|x_{n}-x^{*}\right\|^{2} }
\end{aligned}
$$




$$
\begin{aligned}
& +\alpha_{n}(v-\rho \gamma) \frac{2}{1+\alpha_{n}(v-\rho \gamma)} \frac{1}{v-\rho \gamma} \\
& \times\left(\left\langle\rho U x^{*}-\mu F x^{*}, x_{n+1}-x^{*}\right\rangle+\frac{\beta_{n}}{\alpha_{n}} M\right) .
\end{aligned}
$$

By the conditions (i) and (ii), we have $\Sigma_{n=0}^{\infty} \alpha_{n}(v-\rho \gamma)=\infty$ and

$$
\limsup _{n \rightarrow \infty}\left(\left\langle\rho U x^{*}-\mu F x^{*}, x_{n+1}-x^{*}\right\rangle+\frac{\beta_{n}}{\alpha_{n}} M\right) \leq 0 .
$$

According to Lemma 2.6, we have $x_{n} \rightarrow x^{*}$.

Step 6. In particular, if we take $U=0, F=I$, then $x_{n} \rightarrow x^{*}$, which implies that $x^{*}$ is the minimum norm fixed point of $T$ and $x^{*}$ satisfies the variational inequality (5)

$$
\left\langle(\rho U-\mu F) x^{*}, x-x^{*}\right\rangle \leq 0, \quad \forall x \in \operatorname{Fix}(T) .
$$

So, $\forall x \in \operatorname{Fix}(T)$, we deduce $\left\langle-\mu x^{*}, x-x^{*}\right\rangle \leq 0 \Rightarrow\left\langle x^{*}, x^{*}\right\rangle \leq\left\langle x, x^{*}\right\rangle \leq\|x\|\left\|x^{*}\right\|$, i.e., the point $x^{*}$ is the unique solution to the quadratic minimization problem $x^{*}=\operatorname{argmin}_{x \in \operatorname{Fix}(T)}\|x\|^{2}$. This completes the proof.

Remark 3.1 Prototypes for the iteration parameters in Theorem 3.1 are, for example, $\alpha_{n}=$ $n^{-\xi}, \beta_{n}=n^{-\sigma}$ (with $\xi, \sigma: \frac{1}{2}<\xi<\sigma \leq 1$ ). It is not difficult to prove that the conditions (i)-(iii) are satisfied.

Remark 3.2 Our Theorem 3.1 improves and extends many recent corresponding main results of other authors (see, for example, $[5,13,15-19])$ in the following ways:

(a) Some self-mappings in other papers (see $[15,16,19])$ are extended to the cases of nonself-mappings. Such as the self-contraction mapping $f: H \rightarrow H$ in $[15,16,19]$ is extended to the case of a Lipschitzian (possibly non-self-)mapping $U: C \rightarrow H$ on a nonempty closed convex subset $C$ of $H$. The Lipschitzian and strongly monotone (self-)mapping $F: H \rightarrow H$ in [16] is extended to the case of a Lipschitzian and strongly monotone (possibly non-self-)mapping $F: C \rightarrow H$.

(b) The contractive mapping $f$ with a coefficient $\alpha \in[0,1)$ in other papers (see $[15,16$, $18,19])$ is extended to the cases of the Lipschitzian mapping $U$ with a coefficient constant $\gamma \in[0, \infty)$.

(c) The Mann-type iterative format in $[15-17,19]$ has been extended to the Ishikawatype iterative format (7) in our Theorem 3.1. So, their iterative formats (2), (3), (4) and (6) are some special cases of our iterative format (7), and some of their main results have been included in our Theorem 3.1, respectively.

(d) The iterative approximating fixed point of $T$ in Theorem 3.1 is also the unique solution of the variational inequality (5). In fact, (5) is a hierarchical fixed point problem which closely relates to a convex minimization problem. In hierarchical fixed point problem (1), if $S=I-(\rho U-\mu F)$, then we can get the variational inequality (5). In (5), if $U=0$, then we get the variational inequality $\left\langle F x^{*}, x-x^{*}\right\rangle \geq 0, \forall x \in \operatorname{Fix}(T)$, which just is the variational inequality studied by Suzuki [19]. If the Lipschitzian mapping $U=f, F=I, \rho=\mu=1$, we get the variational inequality $\left\langle(I-f) x^{*}, x-x^{*}\right\rangle \geq 0, \forall x \in \operatorname{Fix}(T)$, which is the variational inequality studied by Yao et al. [18]. So, the results of Theorem 3.1 in this paper have many useful applications such as the quadratic minimization problem $x^{*}=\operatorname{argmin}_{x \in \operatorname{Fix}(T)}\|x\|^{2}$. 


\section{Competing interests}

The authors declare that they have no competing interests.

\section{Authors' contributions}

All authors contributed equally and significantly in this research work. All authors read and approved the final manuscript.

\section{Author details}

'Department of Mathematics, Zhejiang Normal University, Zhejiang, 321004, China. ${ }^{2}$ Department of Mathematics, Tongji Zhejiang College, Zhejiang, 314000, China.

\section{Acknowledgements}

The authors would like to thank editors and referees for many useful comments and suggestions for the improvement of the article. This study was supported by the National Natural Science Foundations of China (Grant Nos. 11271330 11071169), the Natural Science Foundations of Zhejiang Province of China (Grant No. Y6100696).

Received: 24 November 2012 Accepted: 23 April 2013 Published: 7 May 2013

\section{References}

1. Suzuki, T: Fixed point theorems and convergence theorems for some generalized non expansive mappings. J. Math. Anal. Appl. 340, 1088-1095 (2008)

2. Karapinar, E, Tas, K: Generalized (C)-conditions and related fixed point theorems. Comput. Math. Appl. 61(11), 3370-3380 (2011)

3. Karapinar, E, Yuce, IS: Fixed point theory for cyclic generalized weak $\phi$-contraction on partial metric spaces. Abstr. Appl. Anal. 2012, Artticle ID 491542 (2012)

4. Wang, YH: Strong convergence theorems for asymptotically weak G-pseudo- $\psi$-contractive nonself mappings with the generalized projection in Banach spaces. Abstr. Appl. Anal. 2012, Article ID 651304 (2012)

5. Moudafi, A: Viscosity approximation methods for fixed point problems. J. Math. Anal. Appl. 241, 46-55 (2000)

6. $\mathrm{Xu}, \mathrm{HK}, \mathrm{Kim}, \mathrm{TH}$ : Convergence of hybrid steepest-descent methods for variational inequalities. J. Optim. Theory Appl. $119,185-201$ (2003)

7. Matsushita, S, Takahashi, W: A strong convergence theorem for relatively nonexpansive mappings in Banach spaces. J. Approx. Theory 134, 257-266 (2005)

8. Ceng, LC, Cubiotti, P, Yao, JC: Strong convergence theorems for finitely many nonexpansive mappings and applications. Nonlinear Anal. 67, 1464-1473 (2007)

9. Zegeye, $\mathrm{H}$, Ofoedu, EU, Shahzad, N: Convergence theorems for equilibrium problem, variational inequality problem and countably infinite relatively quasi-nonexpansive mappings. Appl. Math. Comput. 216, 3439-3449 (2010)

10. Wang, YH, Xia, YH: Strong convergence for asymptotically pseudo-contractions with the demiclosedness principle in Banach spaces. Fixed Point Theory Appl. 2012, Article ID 45 (2012)

11. Takahashi, W, Wong, NC, Yao, JC: Fixed point theorems and convergence theorems for generalized nonspreading mappings in Banach spaces. J. Fixed Point Theory Appl. 11(1), 159-183 (2012)

12. Chang, SS, Wang, L, Tang, YK, Zhao, YH, Ma, ZL: Strong convergence theorems of nonlinear operator equations for countable family of multi-valued total quasi- $\phi$-asymptotically nonexpansive mappings with applications. Fixed Point Theory Appl. 2012, Article ID 69 (2012)

13. Xu, HK: An iterative approach to quadratic optimization. J. Optim. Theory Appl. 116, 659-678 (2003)

14. Yamada, I: The hybrid steepest-descent method for variational inequality problems over the intersection of the fixed point sets of nonexpansive mappings. In: Butnariu, D, Censor, Y, Reich, S (eds.) Inherently Parallel Algorithms and Optimization and Their Applications, pp. 473-504. North-Holland, Amsterdam (2001)

15. Marino, G, Xu, HK: A general iterative method for nonexpansive mappings in Hilbert spaces. J. Math. Anal. Appl. 318, 43-52 (2006)

16. Tian, M: A general iterative algorithm for nonexpansive mappings in Hilbert spaces. Nonlinear Anal. 73, 689-694 (2010)

17. Ceng, LC, Anasri, QH, Yao, JC: Some iterative methods for finding fixed points and for solving constrained convex minimization problems. Nonlinear Anal. 74, 5286-5302 (2011)

18. Yao, YH, Cho, YJ, Liou, YC: Iterative algorithms for hierarchical fixed point problems and variational inequalities. Math. Comput. Model. 52, 1697-1705 (2010)

19. Suzuki, N: Moudafi's viscosity approximations with Meir-Keeler contractions. J. Math. Anal. Appl. 325, 342-352 (2007)

20. Wang, YH, Yang, L: Modified relaxed extragradient method for a general system of variational inequalities and nonexpansive mappings in Banach spaces. Abstr. Appl. Anal. 2012, Article ID 818970 (2012)

doi:10.1186/1687-1812-2013-121

Cite this article as: Wang and $\mathrm{Xu}$ : Strong convergence of a modified iterative algorithm for hierarchical fixed point problems and variational inequalities. Fixed Point Theory and Applications 2013 2013:121. 